\title{
$k$-anonymity Based Privacy Preserving for Data Collection in Wireless Sensor Networks
}

\author{
K. P. Kaliyamurthie ${ }^{1 *}$, D. Parameswari ${ }^{2}$ and R. Udayakumar ${ }^{3}$ \\ 'Professor \& Head, Bharath University, Chennai-600073; kpkaliyamurthie@gmail.com \\ ${ }^{2}$ Asst. Professor, Dept of MCA, Jerusalem College of Engineering, Chennai; p_kaliyamoorthy@yahoo.com \\ ${ }^{3}$ Associate Professor, Dept. of Information Technology, Bharath University, Chennai-600073; \\ rsukumar2007@hotmail.com
}

\begin{abstract}
In this paper, k-anonymity notion is adopted to be used in wireless sensor networks (WSN) as a security Framework with two levels of privacy. A base level of privacy is provided for the data shared with semi trusted sink and a deeper level of privacy is provided against eavesdroppers. In the proposed method, some portions of data are encrypted and the rest is generalized. Generalization shortens the size of the data transmitted in the network causing energy saving at the cost of information loss. On the other hand, encryption provides anonymization with no information loss and without saving energy. Thus, there is a tradeoff between information loss and energy saving. In our system, this tradeoff is intelligently managed by a system parameter, which adjusts the amount of data portions to be encrypted. We use a method based on bottom up clustering that chooses the data portions to be encrypted among the ones that cause maximum information loss when generalized. In this way, a high degree of energy saving is realized within the given limits of information loss. Our analysis shows that the proposed method achieves the desired privacy levels with low information loss and with considerable energy saving.
\end{abstract}

Keywords: Anonymity, Wireless Sensor Networks, Data Privacy.

\section{Introduction}

Anonymity is defined as a subject being not identifiable within a set of subjects [1]. For many years, the anonymity problem referred to hiding sender and/or receiver identities of messages in data and communication networks. DC-Net and Mix-net solutions were proposed for this aim $[2,3]$. Many other practical anonymity solutions were presented for ISDN networks [4], web and e-mail applications in the Internet [5, 6], for anonymous routing [7], and for ad hoc networks [8]. On the other hand, there are few studies about anonymity in WSNs, mostly based on trying to hide the location or time information of events. The current literature on anonymity in WSNs have several attributes, such as in databases and data mining, the problem of privacy poses several challenges that cannot be easily solved

* Corresponding author:

K. P. Kaliyamurthie (kpkaliyamurthie@gmail.com) by stripping off identity information, due to the fact that other data fields and sources may be jointly used to deduce private information. Suppose several organizations need to share their electronic information, such as public health or demographic data. However, they want to protect the privacy of their consumers or personnel during this information-sharing operation. Simply stripping off the name or social security number from the data set does not solve the privacy problem. It is still possible to identify the owner of a record by using this reduced data in conjunction with other data from various public information sources. This attack technique is called a 're-identification attack'[9] or "record linkage attack" [10]. In order to prevent these type of attacks, Samarati and Sweeney proposed $k$-anonymity [9]. Basically, $k$-anonymity brings a specific restriction to the anonymity problem such that one subject is specifically 
targeted to be hidden among $k-1$ other subjects. In other words, the attributes that may help to identify a subject are modified, via $k$-anonymization, in such a way that each subject has an anonymity set having a size of at least $k-1$. Generally, in privacy problems, the owner of the record or individual having the attributes in the record is assumed to be the subject of anonymity. $k$-anonymization of data is performed by suppression or generalization of some parts. These generalization and suppression operations, however, cause information loss. Thus, it is important to minimize the amount of loss by minimizing the number of suppression and generalization operations, while keeping the data $k$-anonymous. It is shown that achieving optimal $k$-ammonization by minimum number of suppressions is NP-hard even when the alphabet size of attributes equal to three [11].

$k$-anonymity tries to solve the privacy preserving data sharing problem based on a trusted data collection model [10]. In this model, data is collected from data owners by a data publisher. The data publisher releases the data to a data recipient, who analyzes it to complete a required task. A generic example is that of hospitals sharing their medical records with medical research institutions. In this example, the data owners are patients, the data publishers are hospitals, and the data recipients are medical research institutions. Data publishers collect all the details of patient records, but they are required to share them by obeying some privacy criterion. Therefore, patients have to fully trust hospitals in completing privacy preserving operations, but they do not trust the data recipient. Data publishers generally do not know the details of the analysis that is performed by the data recipient, so they share information as much as possible. In the literature, this problem is called "privacy preserving data publishing" [10]. As a result of advances in sensor and wireless technology, wireless sensor networks (WSNs) have emerged as an important information gathering system over wide areas. WSNs have been used to perform critical missions in uncontrolled environments. In some sensor network applications, sensor nodes may deployed over territory that is not physically protected, and may be required to exchange data without a fixed routing structure, gather various types of information from uncontrolled areas, and transfer them to the sinks in controlled or semi-controlled areas. The collected information is not always an aggregated value like average temperature or humidity; it may also be information about specific individuals or events, so that the privacy of each event information becomes important. Moreover, the data gathered in these and other sensor network applications may contain several attributes for an entity. This could enable the launching of re-identification attacks, even in the case where the identities are withheld. Therefore, in such a situation, a $k$-anonymity based solution to provide privacy in WSNs would be more effective.

$k$-anonymity based framework for privacy preserving data..., One of the major threats in wireless mediums is the eavesdropper threat. During the transmission of gathered data to the sink or central server, an adversary having eavesdropping capabilities can sniff the network and obtain event information. Since the sensed area is uncontrolled, the adversary can use his own systems to collect extra information from the area, combine his knowledge with the sniffed event information and thereby determine attributes of specific events, such as location and time. In WSN applications of enemy racing, habitat monitoring, traffic monitoring and human tracking [12], eavesdropping threats have to be dealt with so that the privacy of data during the transmission is ensured. The privacy problem is not limited to the threat of eavesdroppers. In some situations, the data shared with the sink or central server has to fulfill some privacy requirements. There may be a threat of sink capture in some WSN environments, where physical security is not guaranteed. Physical capture of the sink affects the whole system, since it stores all event information. In other applications, the sink itself may not be considered by WNS users to be a fully trustable entity. For example, consider a wireless body area network system where the patient's health status is centrally tracked by a central server (i.e. the sink) in the hospital. Users may not want the central server to know their exact spatio-temporal information unless an emergency occurs. Therefore, privacy of personal information shared with the sink is also needed. As another example, people using WSN applications, such systems caring for the elderly or smart home monitoring systems, may need to protect their privacy from the parties with whom they share personal information. The trusted data collection model used in privacy preserved data publishing methods [10] does not fit directly into WSN environment since the data shared entity, or sink, may not be fully trusted and there may be other un-trusted parties like eavesdroppers. Anonymization must be conducted across multiple trusted entries so that the compromising a trusted entity does not lead to the loss of all the data of the system. Thus, a new data collection model for WSN environments must be be adopted. One of the main design criteria in WSNs is energy consumption, requiring designers of privacy preserved data 
collection systems for WSNs to concentrate on reducing energy costs. Studies show that [13], the energy consumption is heavily dependent on transmission/reception of data packets. Therefore, reducing the size of event information plays a crucial role in energy conservation.

\subsection{Our Contribution}

In this study, we propose a framework for the problem of privacy preserved data collection in WSN applications. Based on the threat model, trusted and un-trusted parties are appropriately chosen among theWSN components and $k$-anonymity is adapted to this environment. As discussed in section 2, our threat model is based on the threat due to untrusted eavesdroppers and semi trusted sinks. Therefore, the model has two privacy criteria: $k 1$-anonymity for the data received by the semi trusted sink and $k 2$-anonymity for the data transmitted in the network that can be captured by the untrusted eavesdropper, where $k 2 \geq k 1$. In this paper, the $k$-Anonymization Clustering Method ( $k$-ACM) is proposed to provide two-layer privacy in WSNs. Our method minimizes the data loss, while keeping the energy consumption within reasonable limits and satisfying the privacy requirements of the threat model. $k$-ACM is based on the unweighted pair group method with arithmetic mean (UPGMA) [14], which is a type of bottom-up clustering method. All anonymization stages are formalized in the bottom-up clustering schema. We perform an information loss calculation, anonymity level measurement and vector distance calculation using the notion of entropy in information theory.

Our method first $k 1$-anonymizes the data to a base level for semi trusted sinks via generalization. Next, the data is further anonymized against eavesdroppers until the data becomes $k 2$-anonymous with the encryption and generalization operations. A trivial solution seems to be totally encrypting the $k 1$-anonymized data. If $k 2$-anonymity is sufficient as a privacy requirement for the eavesdroppers and some amount of data loss can be tolerated by the sink, instead of fully encrypting the $k 1$-anonymous data, this data is $k 2$-anonymized via encryption and generalization operations. In this study, it is shown that this partial encryption method considerably decreases the energy consumption by shortening the lengths of messages. Another contribution of this paper is the presentation of a dynamic taxonomy tree in generalization operations so that smaller amounts of information loss. The rest of the paper is organized as follows. Section 2 presents the details of the network and the threat model of the proposed framework. In Section 3, some basic definitions and background information about UPGMA method and generalization operations with static taxonomy trees are provided.

\section{Network and Threat Model}

Wireless sensor networks are generally deployed in open areas. Third parties can determine some attributes of detected events using their own sensors or by directly observing events. Record linkage attacks may also be conducted to identify the event owner. Our threat model is based on providing privacy by preventing "record linkage attacks" during data collection. Privacy threats are both due to eavesdroppers and the Sink, thus, our threat model addresses the privacy requirements of both of these two threat types. In our model, the privacy requirement levels of the system against the sink and against the eavesdroppers are not the same. We employ a privacy mechanism in which there are two privacy levels associated with the eavesdroppers and sink: untrusted eavesdroppers and semi-trusted sink. Untrusted eavesdroppers are assumed to be capable of capturing data, but the system should ensure that the privacy of the intercepted data is being protected to some extent. In this way, the eavesdroppers can only learn limited information from the intercepted data. On the other hand, while the semi-trusted sink is allowed to legally obtain data, this data should also have a specific privacy property. However, the privacy protection level of the data that the sink obtains is lower than that of the eavesdroppers. In this way, the sink can learn more detailed information in comparison to eavesdroppers, but the detail of the information obtained is still limited. In our network model, there is one sink and a number of sensor nodes. Some sensor nodes serve as aggregation nodes, where all anonymization operations on the data take place. Therefore, our model protects the event data between aggregation nodes and the sink. To prevent "record linkage attack", k-anonymity can be provided by fusing different events. Therefore, trusted entities at which anonymizations can occur must be determined.

\section{Basic Definitions and Background}

In this section, we first explain some basic definitions that are used in the paper. Some background information about static taxonomy trees and the UPGMA algorithm are also given. 


\subsection{Basic Definitions}

Quasi-identifier Attribute: An attribute that is not able to identify a subject by itself, but may help to identify subject with a combination of similar attributes. The set of all quasi-identifier attributes of table $T$ is $Q$.

k-anonymity: Suppose that $T(Q)$ refers to the new table produced by keeping the quasi-identifier attributes and removing the others from table $T$. $T$ has the $k$-anonymity property if and only if each record is indistinguishable from other $k$-1 records in $T(Q)$. Generalization and suppression are more common techniques for making the data $k$-anonymous.

Anonymity Set: If a subject cannot be discriminated from a set of other subjects, the set is called an anonymity set of that subject. In $k$-anonymity, each subject has an anonymity set having at least $k$ - 1 elements.

Generalization: The generalization operation replaces the value of a quasi-identifier attribute with a more general value. For example, a birth date such as ‘04.05.1977’ can be replaced by ' 1977 ' in a generalization operation. Numerical attribute values may also be generalized to numeric intervals.

Suppression: Deletion of a quasi-identifier attribute of a record or removing the whole record entirely. For example, assume that a WSN is constructed for traffic monitoring. This application collects information about the vehicles passing through various locations of the city. Attributes of the sample data are givenon.

\section{Previous Research}

As D. Culler et al. [21] has mentioned in the paper "Overview of Sensor Networks", dense instrumentation, real-time access, and in-network processing make a qualitative difference in our ability to perceive what is happening throughout large physical structures [8]. In environmental monitoring and condition-based maintenance, the purpose of data collection, the parties responsible for using the data, and the scope of dissemination are clear. The situation becomes much less clear in more casual settings in which more general human activity occurs, such as the home, the workplace, a transportation terminal, or a shopping venue. In these cases, many potentially interested parties can have varying uses for the data. More detailed sensing-such as occupancy, motion, and even physiological state-further amplifies concerns over proper use and dissemination. Indeed, image data, such as that obtained by the surveillance cameras in pervasive use today, can be viewed as an extremely powerful sensor, but network access and automated analysis are limited. These social factors are an inherent concern with sensor network technology. Fortunately, this area has become an active focus of research while the technology is still in its early stages.

L. Sweeney [22] has given a solution for formal protection model named k-anonymity and set of accompanying policies for deployment. A release provides k-anonymity protection if the information for each person contained in the release cannot be distinguished from at least $\mathrm{k}-1$ individuals whose information also appears in the release. This paper also examines re-identification attacks that can be realized on release that adhere to k-anonymity unless accompanying policies are respected. The k-anonymity protection model is important because it forms the basis on which the real-world system.

\section{Hypotheses}

\subsection{Taxonomy Trees}

A taxonomy tree is a tree structure that is created for each categorical quasi-identifier attribute to replace an existing attribute value with more general one in the $k$-anonymization process $[19,20,21]$. This replacement is a generalization operation. Leaves of the tree contain distinct values of attributes and nodes in the higher levels of the tree contain more general attribute values. During the anonymization, replacement is done with the values in the higher levels of the tree. There is a root of the taxonomy tree. If the attribute value is generalized up to this point, then the attribute value has no information. Suppression is considered to be another operation for anonymization in the literature [20], but in fact it is a generalization operation where the attribute value is generalized to the root of attribute's taxonomy tree. Consider a sensor network that collects location information, such as address information. A possible taxonomy tree for this location attribute can be constructed as in Figure 3. Suppose that $k$ is chosen as 2 and the location attribute is the quasi-Street.

\subsection{UPGMA Method}

UPGMA [14] The unweighted pair group method with arithmetic mean (UPGMA) is based on the idea of iteratively joining the two closest clusters until one 
cluster is left. A suitable distance definition is required to measure the distance between any two clusters. All distances between each pair of clusters, which are computed according to the distance definition, are stored in a distance matrix at each iteration. At the beginning, each input vector is considered to be an individual cluster. The closest two clusters are found and combined into one common cluster. The distance of the newly formed cluster to the other clusters are recalculated and the distance matrix is updated. This procedure is repeated until one cluster is formed

\section{Research Method}

\section{1 k-anonymization Method}

In this section, our work in $k$-ACM is explained. The first subsection details the ideas behind the proposed dynamic taxonomy tree for generalization methods. The second subsection introduces the information loss metric that is used in selecting the suitable portions for encryption operations and evaluating $k$-ACM results. The third subsection presents the proposed algorithm $k$-ACM. The fourth subsection explains the information theoretic method used to measure the $k$-anonymity level of data. The fifth subsection analyzes the effects of $k$-ACM on the size of data and gives the formulation for saving energy with $k$-anonymization.

\subsection{Generalization Method with Dynamic Taxonomy Tree}

In privacy preserved data collection, the main aim is to share as much as possible with the related parties under the required privacy criterion. Data collection methods generally use static taxonomy trees. Over-generalization is a potential problem of using static taxonomy trees in the generalization of categorical attributes. For example, in the static taxonomy tree given in Figure 3, the generalization of the values 'Buket Street' and 'Selvi street'yields the value 'Istasyon Avenue'. However, this causes information loss, since records containing Istasyon Avenue may also, for example, include 'Serin Street'. To solve this overgeneralization problem as much as possible, we propose using a dynamic taxonomy tree instead of a static one. In the proposed dynamic taxonomy tree model, the tree is dynamically updated by creating new internal nodes (i.e. attribute values) during the generalization and in an ondemand manner, depending on the nature of data and the required generalization. In this method, a new node is generated when the existing parent node has child(ren) other than the generalized nodes. The newly generated node covers the attribute values of the generalized ones only. In this way, generalization is performed with minimum information loss. To perform generalization among any of the attribute values using the proposed dynamic taxonomy tree concept, a flexible data structure is used.

In this data structure, generalizations are implemented by setting the corresponding bits of the attribute values that will be generalized to ' 1 '. Therefore, the total number of bits having the value ' 1 ' increases as generalizations occur. A bit string with many bits of value ' 1 ' actually represents an internal node.

For a numerical attribute, the range of the attribute can be divided into intervals in which each interval has an equal range suitable size. The size of a numerical attribute's bit string is set to the number of intervals. Each interval corresponds to a distinct bit and if an attribute belongs to an interval, the corresponding bit of the interval is set to one in the bit string. The number of intervals can be determined according to the accuracy requirement for that attribute. A higher accuracy requirement implies a larger number of intervals. Increasing the number of intervals enlarges the size of the messages, and thus the required transmission energy. Therefore, a balance between energy and accuracy must be constructed in choosing the number of intervals. Suppose the input data is a table $T$ having $m$ attributes and $n$ records. Tij represents the $j$ 'th attribute of the $i$ th record where $\{i: 1$ $\leq i \leq n\}$ and $\{j: 1 \leq j \leq m\}$. Table $T$ is represented by a set of bit strings $B$, where $B i j$ is the bit string representation of the $j$ 'th attribute of the $i$ 'th record. The $k^{\prime}$ th bit of $B i j$ is written as $B i j(k)$. Suppose that $j$ 'th attribute of the table is categorical and there are $d j$ distinct values. These values are indexed by $k$ and written as $\operatorname{Vj}(k)$, where $\{k$ : $1 \leq k \leq d j\}$. A bit string of this categorical attribute has a size of $d j$ and is formed as follows: If Tij $=V j(k)$ then $\operatorname{Bij}(k)=1$ else Bij $(k)=0$ as $\forall k: 0 \leq k \leq d j$, If an attribute is numerical, the range of the attribute is divided into equal-sized intervals. Assume that the $j$ 'th attribute is numeric and the attribute range is divided into an $e j$ number of intervals. Each interval is indexed by $k$. The bit string representation of this numeric attribute has a size of $e j$ and is formed as follows: If Tij intersects with $k \_$th interval, then Bij $(k)=1$ else Bij $(k)=0$ as $\forall k: 0 \leq$ $k \leq e j$. 
In our proposed model, sensor nodes send their findings directly to aggregation nodes. Aggregation nodes convert quasi-identifier attributes of data to bit strings and $k$-ACM makes them $k$-anonymous. Through the $k$-anonymization process of an attribute, $k$-ACM uses the notion of dynamic taxonomy trees. During the formation of a dynamic taxonomy tree, the bit string of the newly created internal node of a dynamic taxonomy tree is found by the logical OR operation of bit strings of all child nodes.

\subsection{Hypotheses Testing}

\subsubsection{Information Loss Metric}

Calculating the data loss of $k$-anonymous data is needed to predict the performance of our proposed method under different $k$-anonymity parameters. In our study, we use the entropy concept of information theory to measure the information loss [22]. The entropy difference between the $k$-anonymous data and the original data constitutes the information loss. Suppose that $T$ is the input data set having $n$ records and $m$ attributes, $B$ is the bit string representation of this data set, as discussed in Section 4.1, and $C$ is a random variable representing the probability of an attribute value in a $k$-anonymous data entry being the actual attribute value in the original data. Assume that all the entries of $B$ are normalized according to the number of bits having a value of ' 1 ' in that entry (i.e., a 'true bit) and that the normalized version forms the data set $B$. A sample bit string representation set is shown in Table 1. A sample normalized version of the bit string representation set is shown in Table 2.

Information loss of a data table $T, I L(T)$, is equal to the conditional entropy, $H(C \mid B)$. Here, the conditional entropy indicates the uncertainty of the prediction for the original attribute values of a record when we have knowledge of the corresponding $k$-anonymous bit strings

Table 1. A sample bit string representation set

\begin{tabular}{rccc}
\hline Records & Bi1 & Bi2 & Bi3 \\
\hline$T 1$ & 00010 & 01000 & 10000 \\
$T 2$ & 01100 & 11100 & 01111 \\
\hline
\end{tabular}

Table 2. A sample normalized version of the bit string representation set

\begin{tabular}{cccc}
\hline Records & Bi1 & Bi2 & Bi3 \\
\hline$T 1$ & 00010 & 01000 & 10000 \\
$T 2$ & $01 / 21 / 200$ & $1 / 31 / 31 / 300$ & $01 / 41 / 41 / 41 / 4$ \\
\hline
\end{tabular}

of that record. The original data has only one true bit in each bit string, because each original data entry corresponds to one attribute value. However, in $k$-anonymous data, each entry may have more than one attribute value and thus each attribute value is represented by an additional bit. Therefore, if an entry has only one true bit, then it does not have information loss. In this situation, we can be certain that this true bit is the true bit that comes from the original data. As the number of true bits increases, the disorder of the data increases, because it becomes harder to predict which one is the original true bit. Prediction gets harder because information is lost as a result of the increase in the number of true bits. Conditional entropy, which is used to calculate the disorder of the data, is a good measurement tool for information loss. Conditional entropy $H(C \mid B)$, which is equal to the information loss of table $T, I L(T)$, can be found as follows:

$$
\begin{aligned}
& I L(T)=H(C \mid B)={ }_{-} B i j \in B p(B i j) H(C \mid B=B i j)= \\
& { }_{{ }_{-}} B i j \in B p(B i j){ }_{k} \in\{1 . . z\} p(C=k \mid B i j) \log p(C=\mid B i j)
\end{aligned}
$$

In Eq. (1), it is assumed that each attribute is converted to bit strings having size $z$. This implies that all categorical attributes have $z$ distinct attribute values and all numerical attributes have $z$ number of interval ranges. Also, it is assumed that all $k$ 's, where the equalities of $p(C=k \mid$ $B i j)=0$ are true, are excluded from the summation. The random variable $C$ can take on values from the set $\{1 . . z\}$. In fact, $B$ is calculated for finding the value of this random variable.

$p(C=k \mid B=B i j)=B i j(k)$ for each $k: 1 \leq k \leq z$

In Eq. (1), it is assumed that each record has an equal probability of being chosen and that each attribute of the record has the same probability. Therefore, the probability mass function of the $j$ 'th attribute of the $i$ 'th record, $p(B i j)$, is calculated as

$$
p(B i j)=1 \text { m.n. }
$$

Eq. (1) can be rewritten as follows:

$$
\begin{aligned}
& I L(T)=H(C \mid B)=-_{-} B i j \in B 1 m . n_{-} k \in 1 . . z \\
& B i j(k) . \log B i j(k)
\end{aligned}
$$

Suppose that $F$ is the array that contains the number of true bits of the bit string array $B$. The total number of true bits in Bij is Fij. The total number of elements in $B i j(k)$ that has the value $1 F i j$ is equal to $F i j$, and the rest is zero. Therefore, the second summation operation of Eq. (3) yields the value log 1 Fij. The simplest equation for the information loss of the data table $T, I L(T)$, can be calculated as follows: 
$I L(T)=H(C \mid B)={ }_{-}$FijeF 1 m.n $\log 1$

$F i j=1 m . n_{-} F i j \in F \log F i j$

\subsection{Clustering Algorithm for $k$-anonymization}

$k$-anonymization produces an output such that the number of any quasi-attribute value set is not less than $k$. An optimal method must find groups of records possessing this property with minimum data loss. Since this problem has been shown to be an NP-Hard problem [11,23], various heuristic methods have been developed to prevent data loss as much as possible $[9,21]$. In this paper, we adapt a bottom-up hierarchical clustering technique to the $k$-anonymization problem. The proposed method is called the $k$-anonym Clustering Method ( $k$-ACM). The clustering algorithm is applied to the portion of the data containing only quasi-identifier attributes. The basic idea is to partition the data vectors into clusters, where each cluster has at least $k$ vectors. After clustering, the vectors in one cluster are anonymized to a common vector. Each cluster forms a representative vector that is actually the $k$-anonymization output of all vectors in that cluster. All quasi-identifier attributes of the input data are replaced with the corresponding attributes of the representative vector. This clustering process ensures that similar vectors are grouped in clusters so that their anonymization does not cause significant data loss. The run time of $k$-ACM is found to be $O(n 2 \log n)$, where $n$ is the number of event records.

\subsection{Method of Distance Calculation}

The aim of our method is to minimize information loss, while providing the required level of $k$-anonymity. Ateach iteration of $k$-ACM, two clusters are combined. Each cluster combination leads to some generalization operations and therefore to information loss. $k$-ACM should choose the most suitable cluster pair that creates the least information loss when combined. To do so, a suitable distance calculation method is required. In Section 4.2, the notion of conditional entropy is used to calculate the overall information loss of $k$-anonymized data. This notion is adapted to calculate the distance between any two clusters as the entropy loss caused by merging them. At the hth iteration, the distance between the sth and $t$ th clusters is defined as $D h[s][t]$. Suppose that the cluster resulting from merging the clusters $s$ and $t$ is represented as cluster $u$ in iteration $h+1$.

\section{The Results of Hypotheses Testing}

\subsection{Performance Evaluation of k2- anonymization Stage}

The $k 2$-anonymization stage starts with the $k 1$-anonymous data obtained from the previous stage. The output enlargement factor, $M$, adjusts the length of the output that is obtained at the end of the $k 2$-anonymization stage. As $M$ increases, the size of the output, and, consequently, the number of encrypted data entries, increases as. This increase yields a more accurate output and decreases the amount of information loss. Table 3 , shows experimental results for a data set having 500 records, where $k 1=4$ and $k 2=16$. The first column lists the value of the output enlargement factor, $M$, that is pre-determined for the corresponding experiment. The second column shows the anonymity level that the output provides and is computed according to Eq. (10). This anonymity level is obtained after the encrypted parts are decrypted at the sink. The third column gives the information loss incurred in $k 2$-anonymization stage alone, and is the difference between the information loss incurred at the end of the $k 2$-anonymization stage and at the end of $k 1$-anonymization stage.

In this research, we investigate companies listed in Tehran Stock Exchange and state companies. We study the companies listed in TSE because of, ease of access to the data of these companies, as wall as, regulations of the TSE cause the more harmonious financial statements information. And, we study state companies because of ease of access to their data throughout the Accounting Practices and Accounts Consolidation Agency. All state companies required to send their financial statements to this Agency. The other reason for studying the state companies is the frequency of fixed assets revaluations, and foreign currency adjustments (other comprehensive income items based on Iranian GAAP). The Iranian companies have been required to issue the comprehensive income statement since 2001. At the time of this study the financial statements for three years are available, therefore, the time period of the study restricted to 2001-2003.

Table 3. Experimental results of data set with 500 records for the $\mathrm{k} 2$-anonymization part

\begin{tabular}{cccc}
\hline $\begin{array}{l}\text { Output } \\
\text { Enlargement Ratio }\end{array}$ & $\begin{array}{c}\text { Anonymity } \\
\text { Level }\end{array}$ & $\begin{array}{c}\text { Information } \\
\text { Loss }\end{array}$ & $\begin{array}{c}\text { Energy } \\
\text { Saving }\end{array}$ \\
\hline 4.80 & 4.88 & 0.95 & 0.76 \\
0.25 & 3.72 & 0.54 & 0.25 \\
0.50 & 3.07 & 0.29 & 0.50 \\
0.75 & 2.67 & 0.13 & 0.75 \\
1.00 & 2.25 & 10.00 & 1.00 \\
\hline
\end{tabular}


7.2 Sample

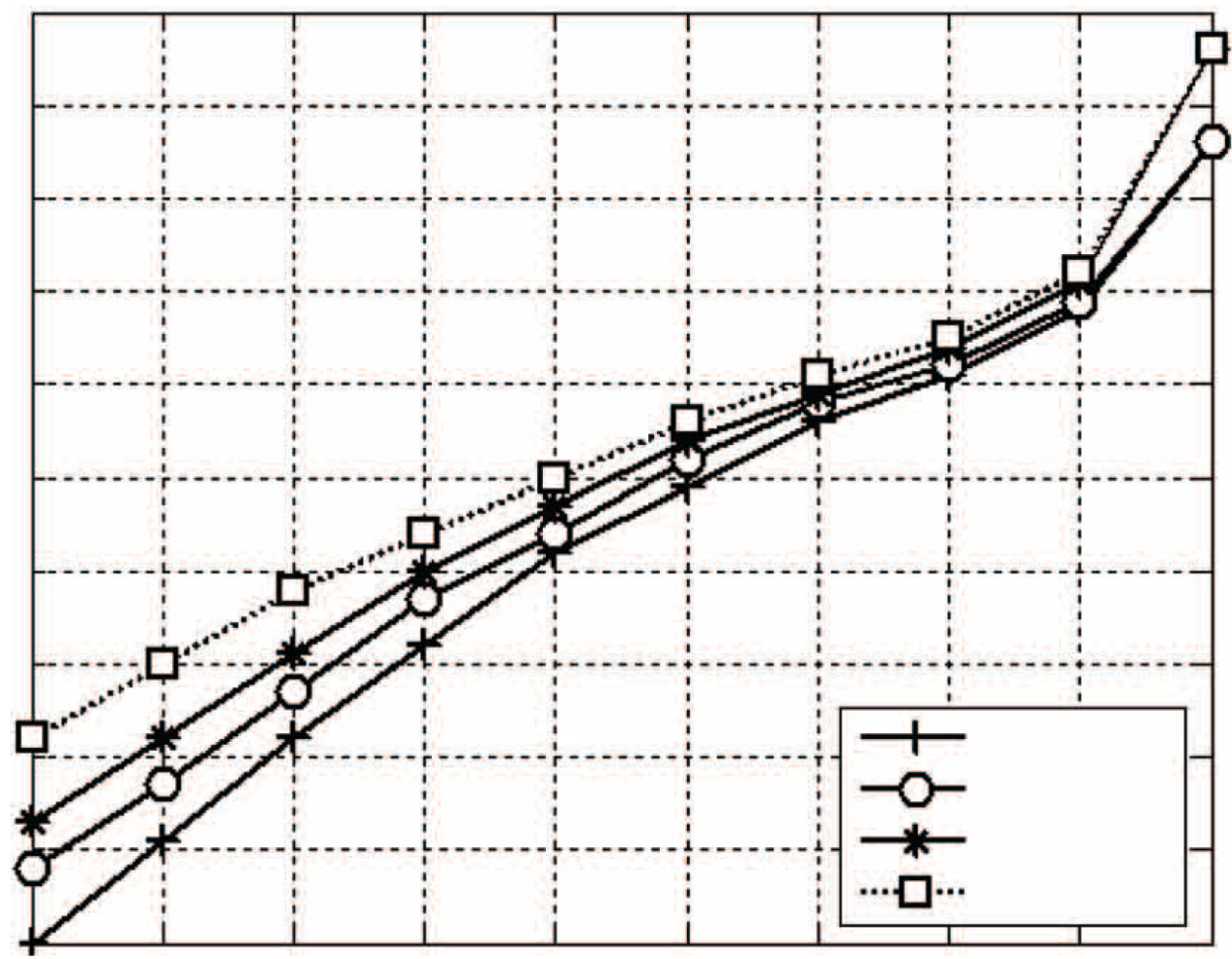

Output enlargement factor vs. energy savings in the $k 2$-anonymization stage

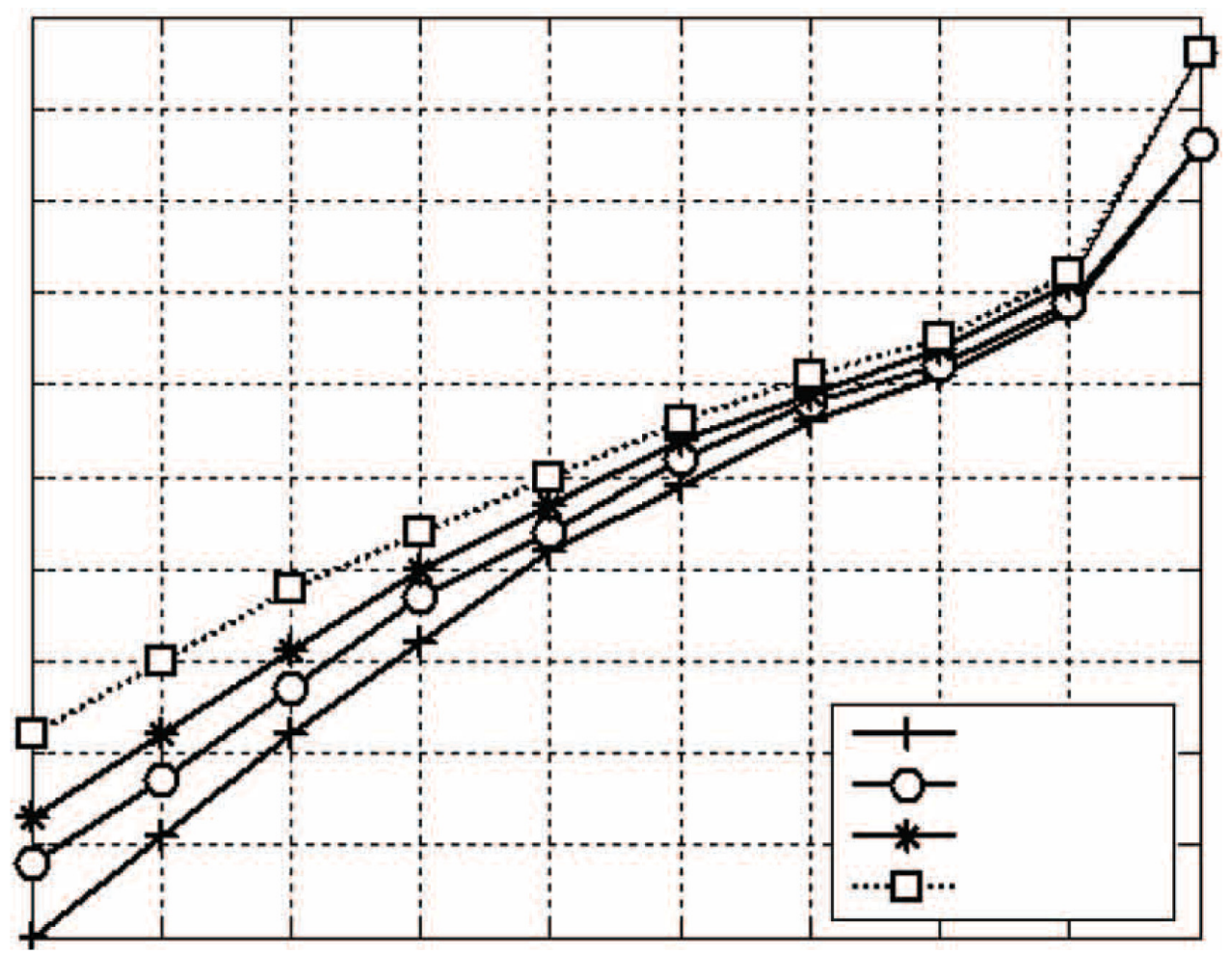

Energy saving/information loss for different $M$ values at the $k 2$-anonymization stage 


\section{Related Work}

Anonymity solutions in the literature are mainly based on two theoretical studies: DC-Nets and mixes [2,3]. The basic idea behind DC-Nets is to anonymously broadcast a message to ensure receiver anonymity.

If the message is intended to be sent to a specific destination, DC-Nets use secret and public key cryptography. However, DCNets only address the problem of recipient anonymity; sender anonymity is not considered. Performing secret key and public key distribution can also be difficult and inefficient in large networks $[18,19]$. Mixes is an important idea for providing sender-recipient anonymity. In mixes solutions, nodes iteratively encrypt the message at each hop and relay the entire message to the next hop. No eavesdropper will be able to deduce the destination and source information from the content of the message. The mixes concept is used in practical applications, such as ISDN networks [4], e-mail and web applications [5, 6]. Mobile ad hoc networks benefit from anonymous routing protocols in providing sender-receiver anonymity $[7,8]$ by adapting Mix-net ideas. Since every node behaves as a router in these networks, the exchange of routing information comprises a considerably important amount of the legitimate traffic. Malicious users can deduce the sender and receiver of a message from the routing messages. To the best of our knowledge, there are few studies about the anonymity problem in WSNs, most of which attempt to hide information about the location or time of events. Gruteser, et. al. [19, 20] proposed anonymity solutions for providing a high degree of privacy in sensor networks for location-based services, such as traffic monitoring, fleet management and 'pay as you drive' insurance. They claim that adversarial eavesdropping on event messages can identify a specific individual by linking the event location information with apriori knowledge about the event. In this study, however, the location and time information of events are cloaked so that such an outsider cannot differentiate any individual from among the other $\mathrm{k}$ different individuals. Ozturk et al. proposed a phantom routing method for hiding location information of originator sensor nodes in a sensor network geared towards tracking moving objects. The threat model is based on the existence of only one movable adversary node in the environment. The adversary tries to reach a moving target object by eavesdropping on the traffic and finding the previous hop of routing messages $[9,10]$. The routing algorithm proposed aims to make such catching operations difficult by hiding the location of sensor nodes[13,14].
The proposed routing protocol prevents the eavesdropper from identifying the receiver by tracing the wireless packets[11,12]. It randomizes the routing paths and injects fake packets in order to mislead eavesdroppers. Wadaa et al. studied providing anonymity of the coordinate system, cluster and routing structures during the network setup of a WSN. Castelluccia et al. proposed a homomorphic encryption method that securely aggregates sensor findings in an energy efficient way. This work deals with the aggregation functions, which compute the average or variance of sensor findings. Protection of location privacy is guaranteed by $k$-anonymity in location-based services that are given on mobile networks [17]. In this work, each mobile client specifies a minimum level of anonymity, and maximum temporal and spatial tolerances.

The proposed methods try to provide the needed anonymity level within these qualities of service parameters. Privacy issues are becoming more and more important for data mining applications in which organizations share information with each other. Privacy preserving methods in data mining applications are explored in some database studies $[15,16]$. These studies use perturbation methods on confidential parts of data. In this way, the adversary can obtain only perturbed data that has different distribution characteristics. Perturbation is done so that the application of data mining techniques to perturbed data will result the same accurate mining results. Generally, the organization who shares its data does not know the details of the analysis or data mining tasks which will be performed by data recipient party.

\section{Summary and Concluding Remarks}

In this paper, we proposed a $k$-anonymization clustering method ( $k$-ACM) that provides a $k$-anonymity framework for WSNs. Our threat model implies two levels of $k$-anonymity: 1) against the semi trusted sink, and 2) against eavesdroppers. Our threat model assumes the sink is a semi trusted entity, so that the data received by the sink must be at least $k 1$-anonymous. To protect the data against eavesdroppers, data transmitted through the network must be at least $k 2$-anonymous. Since the minimum protection against eavesdroppers must be greater than the minimum protection against the semi trusted sink, $k 2$ is greater than $k 1$. designers can decide on the values of $k 1$ and $k 2$ by considering the security threats of the environment and application requirements. For example, military WSN applications can use higher $k 1$ and $k 2$ values during times of in war, and 
lower values during times of peace. If the possibility of an eavesdropper threat is high and the security of the sink is provided, then the WSN designers can choose higher $k 2$ and lower $k 1$ values. In $k$-ACM, there is a tradeoff between data quality (in terms of information loss) received at the sink and energy consumption. The quality of data is reduced by generalization, since that operation irreversibly perturbs the data, thereby causing information loss. $k$-ACM first makes the data $k 1$-anonymous by applying generalization operations and continues to implement $k 2$-anonymization via generalization and encryption operations. The output size of the $k 2$-anonymization stage can be adjusted by a predetermined threshold value, or output enlargement factor. As this ratio increases, the size of the output gets larger and more encryption operations are performed. During this enlargement, $k$ - ACM selects the most suitable portions of the data for encryption to minimize information loss as much as possible. An increase of the output enlargement factor causes an increase in the energy consumption in the WSN. On the other hand, the quality of data received at the sink improves since the data is not greatly perturbed. If the ratio decreases, the quality of data also decreases, but the system consumes a considerably smaller amount of energy. In fact, $k$-ACM provides a mechanism for WSN designers to balance between information loss and energy cost by using the output enlargement factor. Our analyses show that the energy savings per information loss value constantly increases as the output enlargement factor increases. This implies that WSN designers should pick the maximum output enlargement factor that the information loss and/or energy saving restrictions of the WSN dictate. For example, our analysis shows that for a sink that is to receive 4-anonymous data (i.e. $k 1=4$ ) and 12 -anonymous data, which are required against eavesdroppers (i.e. $k 2=12$ ), if the network can tolerate an information loss of entropy value 0.37 , then WSN designers can pick an output enlargement factor of 0.4 , resulting in an energy savings of $43 \%$ energy while also providing the required anonymity levels. $k$-anonymization of data is performed by an algorithm that is based on UPGMA, a well-known bottom up hierarchical clustering mechanism. The notion of conditional entropy from information theory is adapted for use as a distance function, which calculates the information loss during each clustering process. The same notion is also used for calculating the anonymity level of $k$-anonymous data and for evaluating the results of experiments in terms of information loss. Additionally, this paper introduces a dynamic taxonomy tree concept for the generalization operation.

\section{References}

1. Pfitzmann A, and Khntopp M (2000). Anonymity, unobservability, and pseudonymity - A proposal for terminology, Designing Privacy Enhancing Technologies, Lecture Notes in Computer Science, vol 2009/2001, 1-9.

2. Chaum D (1988). The dining cryptographers problem: Unconditional sender and receipent untraceability, Journal of Cryptology, vol 1(1), 65-75.

3. Chaum D (1981). Untraceable electronic mail, return addresses, and digital pseudonyms, Communications of the Associations for Computing Machinery, vol 24(2), 84-88.

4. Pfitzmann A, Pfitzmann B et al. (1991). ISDN-Mixes: untraceable communication with very small bandwidth overhead, Proceedings of the GI/ITG Conference on Communication in Distributed Systems, 451-463.

5. Gulcu C, and Tsudik G (1996). Mixing e-mail with BABEL, Symposium on Network and Distributed Systems Security (NDDS '96).

6. Reiter M K, and Rubin A D (1999). Anonymous web transactions with crowds, Communications of the ACM, vol 42(2), 32-48.

7. Reed M G, and Syverson P F (1998). Anonymous connections and onion routing, IEEE Journal on Selected Areas in Communications, vol 16 (4), 482-494.

8. Kong J, Hong X et al. (2003). An anonymous on demand routing protocol with untraceable routes for mobile ad hoc networks, Proceedings of the 4th ACM International Symposium on Mobile Ad Hoc Networking \& Computing, 291-302.

9. Samarati P, and Sweeney L (1998). Generalizing data to provide anonymity when disclosing information, Proceedings of the $17^{\text {th }}$ ACM SIGMOD-SIGACT-SIGART Symposium on the Principles of Database Systems.

10. Fung B C M, Wang K et al. (2009). Privacy-preserving data publishing: a survey on recent developments, ACM Computing Surveys.

11. Meyerson A, and Williams R (2004). On the complexity of optimal k-anonymity, Proceedings of the 23rd ACM SIGMOD-SIGACTSIGART Symposium on the Principles of Database Systems, 223-228.

12. Yick J, Mukherjee B et al. (2008). Wireless sensor network survey, Computer Networks, vol 52(12), 2292-2330.

13. Carman D W, Kruus P S et al. (2000). Constraints and approaches for distributed sensor network security, NAI Laboratories, Technical Report 00-010.

14. Michener C D, and Sokal R R (1957). A quantitative approach to a problem in classification, Evolution, vol 11, No. 2, 130-162.

15. Chan H, Perrig A et al. (2003). Random key predistribution schemes for sensor networks, Proceedings of 2003 Symposium on Security and Privacy, 197-213. 
16. Du W, Deng J et al. (2005). A pairwise key predistribution scheme for wireless sensor networks, ACM Transactions on Information and System Security (TISSEC), vol 8(2), 228-258.

17. Heinzelman W, Kulik J et al. (1999). Adaptive protocols for information dissemination in wireless sensor networks, Proceedings of the 5th Annual ACM/IEEE International Conference on Mobile Computing and Networking (MobiCom'99).

18. Intanagonwiwat C, Govindan R et al. (2000). Directed diffusion: a scalable and robust communication paradigm for sensor networks, Proceedings of the 6th Annual ACM/ IEEE International Conference on Mobile Computing and Networking (MobiCom'00).
19. Fung B C M, Wang K et al. (2005). Top-down specialization for information and privacy preservation, Proceedings of the 21st International Conference on Data Engineering, 205-216.

20. Sweeney L (2002). Achieving k-anonymity privacy protection using generalization and suppression, International Journal of Uncertain, Fuzziness Knowledge-Based Systems, vol 10(5), 571-588.

21. Culler D, and Estrin M S D (2004). Overview of Sensor Network, Computer, vol 37(8), 41-49.

22. Sweeney L (2002). A model for protecting privacy, International Journal on Uncertainty Fuzziness and Knowledge-based System, vol 10(5), 557-570. 